\title{
Comparison of Radiobiological Models for Radiation Therapy Plans of Prostate Cancer: Three-dimensional Conformal versus Intensity Modulated Radiation Therapy
}

\author{
Mesbahi A. ${ }^{1,2,3 * \odot}$, Rasouli N.1,2, Mohammadzadeh M.3, \\ Nasiri Motlagh B. ${ }^{3,4}$, Ozan Tekin H. ${ }^{5}$
}

\begin{abstract}
Purpose: In the current study, using different radiobiological models, tumor control probability (TCP) and normal tissue complication probability (NTCP) of radiotherapy plans were calculated for three-dimensional conformal radiation therapy (3D-CRT) and intensity modulated radiation therapy (IMRT) of prostate cancer.

Methods and Materials: 10 prostate plans were randomly selected among patients undergoing radiation therapy of prostate cancer. For each patient, 3D-CRT and IMRT plans were designed to deliver, on average 76 Gy and 82 Gy to planning target volume, respectively. Using different radiobiological models including Poisson, equivalent uniform dose (EUD) and Lyman-Kutcher-Burman (LKB), TCP and NTCP were calculated for prostate and critical organs including bladder, rectum and femoral heads.
\end{abstract}

Results: IMRT plans provided significantly lower NTCP for bladder, rectum and femoral heads using LKB and EUD models ( $p$-value $<0.05$ ). The EUD-calculated TCP for prostate cancer revealed no considerable improvement for IMRT plans relative to 3D-CRT plans. However, the TCPs calculated by Poisson model were dependent on $\alpha / \beta$, and higher TCP for IMRT relative to 3D-CRT was seen for $\alpha / \beta$ higher than 5.

Conclusion: It can be concluded that IMRT plans were superior to 3D-CRT plans in terms of estimated NTCP for studied critical organs. On the other hand, different mathematical models provided different quantitative outcome for TCP of prostate cancer plans. More clinical studies are suggested to confirm the accuracy of studied radiobiological models.

Citation: Mesbahi A, Rasouli N, Mohammadzadeh M, Nasiri Motlagh B, Ozan Tekin H. Comparison of Radiobiological Models for Radiation Therapy Plans of Prostate Cancer: Three-dimensional Conformal versus Intensity Modulated Radiation Therapy. J Biomed Phys Eng. 2019;9(3):267-278. https://doi.org/10.31661/jbpe.v9i3Jun.655.

\section{Keywords}

Tumor Control Probability, Normal Tissue Complication Probability, Radiobiological Modelling, IMRT, 3D-CRT, Radiation Therapy, Prostate Cancer

\section{Introduction}

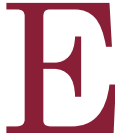

valuation of radiation therapy plans using the quantitative criteria to select optimum irradiation technique plays a vital role in the outcome of radiation treatments. There are two standard and well-established indicators, dose distribution and dose volume histogram (DVH) which serve to differentiate between available treatment configurations to achieve the most desirable clinical outcome. Nevertheless, radiobiological modelling was added to some treatment plan-

*Corresponding author: A. Mesbahi,

Ph.D. Medical Physics Department, Medical School, Attar Street.

Tabriz, Iran

E-mail: amesbahi2010@ gmail.com

Received: 24 September 2016 Accepted: 8 December 2016 
ning systems (TPSs) to help plan evaluation as well as to create radiobiological index for every plan and dose distributions [1-5]. Biological modelling basically utilizes the DVH of a given plan and biological parameters of tumor type and normal critical tissues for the calculation of tumor control probability (TCP) and normal tissue complication probability (NTCP) [4, 6-8]. There are several studies on the application of radiobiological modelling for treatment plans and ranking the rival treatment plans for different radiation treatments [1-3, 9-12]. Several investigations have shown that radiobiological ranking of treatment plans helps clinicians find optimum treatment techniques when the physical dose distribution and DVH results are very close and very hard to differentiate $[2,3,13,14]$; for instance, Luxton et al. compared TCP and NTCP for prostate cancer patients planned for three-dimensional conformal radiotherapy treatments (3D-CRT) and intensity modulated radiotherapy (IMRT) [3]. A greater TCP was found for IMRT relative to 3D-CRT. For all studied organs, mean NTCP was lower for IMRT than for 3D-CRT. In a study by Deb et al., 3D-CRT and IMRT treatments of the prostate were compared using a radiobiological model [12]. They recommended more clinical studies to refine the radiobiological models and related parameters. In another study, a comparison was performed between 3D-CRT and IMRT plans for postprostatectomy radiation therapy. Bladder and rectal doses were lowered with IMRT planning in comparison to $3 \mathrm{D}$ conformal planning [2]. However, in spite of the dosimetric differences between two methods, radiobiological modelling estimated non-significant differences in the NTCP of both bladder and rectum.

In the current study, we used different available radiobiological models for TCP/NTCP calculations to investigate the differences between studied radiobiological models in 3DCRT and IMRT plans. Moreover, a new treatment planning system (TPS), TiGRT was used for 3D-CRT and IMRT planning of prostate cancer patients. The purpose was to validate the planning results by comparison of 3DCRT and IMRT plans in terms of radiobiological metrics, TCP/NTCP.

\section{Material and Methods}

\section{Study Design}

We performed a retrospective study on ten intact prostate cancer patients (intermediate and high risks) which had been treated with 3D-CRT technique in radiation oncology department of Tabriz International Hospital. The patients were selected randomly among available plans in our treatment planning system. One physician, radiation oncologist, supervised planning and performed contouring for all prostate plans.

Tumor volumes and critical organs were outlined slice by slice on the patient's x-ray computed tomography datasets. The clinical tumor volume (CTV) including prostate, seminal vesicles and the surrounding organs at risk including bladder, rectum, right and left femur heads were outlined.

For 3D-CRT plans, planning target volume (PTV) included prostate clinical volume plus $5 \mathrm{~mm}$ margin and seminal vesicles which received $76 \mathrm{~Gy}$, and the left and right lymph nodes were planned to receive the average dose of 50 Gy. The 3D-CRT plans were divided into two courses including an extended field with 50 Gy covering the whole pelvis for lymphatic involvements. Then, in the second course, 6 conformal beams were used to deliver the dose of 26 Gy to the prostate and seminal vesicles. All beams were planned with $18 \mathrm{MV}$ photons.

IMRT plans were generated for each case of ten patients using TiGRT TPS (LinaTech, Sunnyvale, CA, USA) a commercial planning system. The TPS was evaluated by previous studies for its accuracy in 3D-CRT and IMRT calculations $[15,16]$. IMRT plans were generated after choosing beam angles that helped to limit dose to the organs at risk and reduced 
optimization time. All beam energies were 18 MV and shaped with MLCs of Siemens-Oncor linac with 41 pairs leaves. Each leaf had a thickness of $1 \mathrm{~cm}$ at isocenter. The IMRT technique consisted of an arrangement of 7 coplanar intensity-modulated beams. A step and shoot module was used for the following fixed gantry angles: $0^{\circ}, 52^{\circ}, 103^{\circ}, 154^{\circ}, 205^{\circ}$, $256^{\circ}$ and $308^{\circ}$. The inverse planning procedure was performed with TiGRT planning. The PTV was prescribed to a mean dose of 82 Gy by 41 fractions. PTV was planned to be encompassed by $95 \%$ isodose. The resulting set of dose requirements for the targets is summarized in Table 1 along with dose-volume constraints used for the critical organs.

\section{TCP and NTCP Calculations using Bioplan Software}

For biological evaluation of our plans, we used Bioplan (ver.1.3.3) software developed by Sanchez-Nieto and Nahum [17]. The software was provided freely by developers on a personal request. Differential dose volume histograms (dDVHs) were computed for the CTV, bladder, rectum and femoral heads. DVHs were exported as ASCII files for Bioplan with a dose bin width of $0.25 \mathrm{~Gy}$, corresponding to a total of approximately 333 bins; volumes were given in $\mathrm{cm}^{3}$. TCP was calcu- lated for $\alpha / \beta=1.5,3,5$ and 10 in each plan using the Poisson model; we know the $\alpha / \beta$ ratio indicates the sensitivity of a tissue to fractionation. The calculation was based on the CTV which received dose distribution. A default set of model parameters for prostate was taken from Bioplan. Additionally, the NTCP was estimated from the Lyman-Kutcher-Burman (LKB) model [17-19]. The NTCP calculation in LKB model is defined as:

$$
\begin{gathered}
\operatorname{NTCP}(D, v)=\frac{1}{\sqrt{2 \pi}} \int_{-\infty}^{t} \exp \left(-\frac{1}{2} x^{2}\right) d x \\
\mathrm{t}=\frac{D-T D_{50}(v)}{m \cdot T D_{50}(v)} \\
T D_{50}(v)=\frac{T D_{50}(1)}{v^{n}}
\end{gathered}
$$

Where $\mathrm{TD}_{50}(v)$ is the tolerance dose for a $50 \%$ complication probability caused by uniform irradiation to $\mathrm{v}$. $\mathrm{n}$ is the volume exponent and $\mathrm{m}$ is a parameter, which is inversely related to the steepness of the dose-response curve. Specific complication endpoints for rectum (severe proctitis-necrosis-stenosis-fistula and mild RTOG grade $2 / 3$ ), bladder (symptomatic bladder contracture and volume loss) and femoral heads (necrosis) were selected. The corre-

Table 1: Dose specifications and constraints used for IMRT planning.

\begin{tabular}{cc} 
Structure & constraint \\
\hline Rectum & $\mathrm{V}_{70 \mathrm{~Gy}}=25 \%$ \\
\hline Dorsal part of rectal wall & $\mathrm{D}_{\max } \leq 65 \mathrm{~Gy}$ \\
\hline Bladder & $\mathrm{V}_{70 \mathrm{~Gy}}=50 \%$ \\
\hline Femoral heads & $\mathrm{V}_{52 \mathrm{~Gy}}=10 \%$ \\
\hline CTV & 77.9 Gy $\leq \mathrm{DCTV} \leq 86.1 \mathrm{~Gy}$ \\
\hline PTVCTV & $70.1 \mathrm{~Gy} \leq \mathrm{DPTVCTV} \leq 77.9 \mathrm{~Gy}$ \\
\hline PTV-Rectum overlap & Dmax not within this region
\end{tabular}

Abbreviations: $V x G y$ - percentage of structure volume receiving $X$ Gy or more; Dmax: depth of dose maximum 
sponding set of parameters for TD50, n, and $\mathrm{m}$ consisted of $80,0.5$ and 0.11 for bladder; 80 , 0.12 and 0.15 for rectum severe proctitis; 56 , 0.203 and 0.064 for rectum mild RTOG grade $2 / 3$ complication; and finally $56,0.25$ and 0.12 for femoral heads. These parameters were derived from different studies $[13,18]$ and used recently by Jensen et al. [1]

\section{Calculation by Equivalent Uniform Dose Model}

For calculations using equivalent uniform dose (EUD) model, cumulative DVHs of plans (3D-CRT and IMRT) were exported from TiGRT TPS. We used a Matlab m-file to calculate the Niemierko's equivalent uniform dose, EUD-based NTCP-TCP values [20].

According EUD model, for a dose of $2 \mathrm{~Gy}$ in each fraction the EUD, equivalent dose (EQD), TCP and NTCP were calculated by the following equations:

$$
\begin{gathered}
E U D=\left(\sum_{i=1}\left(v_{i} E Q D_{i}^{a}\right)\right)^{\frac{1}{a}} \& E Q D=D \times\left(\frac{\frac{\alpha}{\beta}+\frac{D}{n_{f}}}{\frac{\alpha}{\beta}+2}\right) \\
\mathrm{TCP}=\frac{1}{1+\left(\frac{T C D_{50}}{E U D}\right)^{4 \gamma_{50}}} \\
\mathrm{NTCP}=\frac{1}{1+\left(\frac{T D_{50}}{E U D}\right)^{4 \gamma_{50}}}
\end{gathered}
$$

In all above equations, $a$ is a unit-less model parameter for each normal structure or tumor of interest, and $v_{i}$ is unit-less and represents the $i^{\text {th }}$ partial volume receiving dose $D_{i}$ in Gy. $n \mathrm{f}$ and $\mathrm{df}=\mathrm{D} / n \mathrm{f}$ are the number of fractions and dose per fraction size of the treatment course, respectively. The $\alpha / \beta$ is the tissue specific Linear Quadratic (LQ) parameter of the organ being exposed. $\mathrm{TCD}_{50}$ is the tumor dose to control $50 \%$ of the tumors when the tumor is homogeneously irradiated, and the $\gamma 50$ is a unit-less model parameter that is specific to the tumor of interest and describes the slope of the dose response curve, and $\mathrm{TD}_{50}$ is the tolerance dose for a $50 \%$ complication rate at a specific time interval when the whole organ of interests were homogeneously irradiated. Radiobiological parameters of Burman et al. [18] were used for EUD model calculations as summarized in Table 2.

\section{Results}

The mean dose for all studied volumes including prostate and organs at risk were obtained from treatment plans. Bladder, rectal and femoral heads sparing improved with IMRT planning compared to 3D-CRT planning. The respective average mean doses (Gy) for 3D-CRT and IMRT plans were 76.38 and 82.47 for prostate, 60.72 and 53.5 for rectum, 62.63 and 54.08 for bladder, for 49.67 and 39.94 femoral heads.

In Figure 1, the dose distribution and DVH

Table 2: Parameters used to calculate Niemierko's EUD-based TCP for prostate cancer and NTCP for rectum, bladder and femoral heads.

\begin{tabular}{ccccccc} 
Tissue & $\mathbf{a}$ & Y50 & TD50(Gy) & TCD50(Gy) & Dpf (Gy) & $\boldsymbol{\alpha} / \boldsymbol{\beta}(\mathbf{G y})$ \\
\hline Prostate & -10 & 1.0 & - & 28.34 & 2 & 1.20 \\
\hline Rectum & 8.33 & 4 & 80 & - & 2 & 3.90 \\
\hline Bladder & 2 & 4 & 80 & - & 2 & 8.00 \\
\hline femur heads & 4 & 4 & 65 & - & 2 & 0.85
\end{tabular}

Dpf:dose per fraction, EUD: equivalent uniform dose, TD: tolerance dose, TCD: tumor dose to control 
comparison have been demonstrated for patient No.3. It should be mentioned here that the prescription doses of 76 and 82 Gy for PTV were used for 3D-CRT and IMRT plans, respectively.

In Table 3, the NTCP of rectum for all patients was calculated by using Bioplan software and LKB model. The $\alpha / \beta$ ratio of 3 was used for calculations. Two types of complications including proctisits/necrosis/stenosis/ fistula and RTOG 2/3 mild complication were estimated. Table 3 shows that the probability of mild rectal complication is significantly higher (almost 10 times) relative to proctisits/ necrosis/stenosis/fistula complications. Furthermore, the average NTCP for both complications were higher for 3D-CRT relative to IMRT plans. However, the difference between 3D-CRT and IMRT became pronounced for mild RTOG2/3 complication. The paired t-test

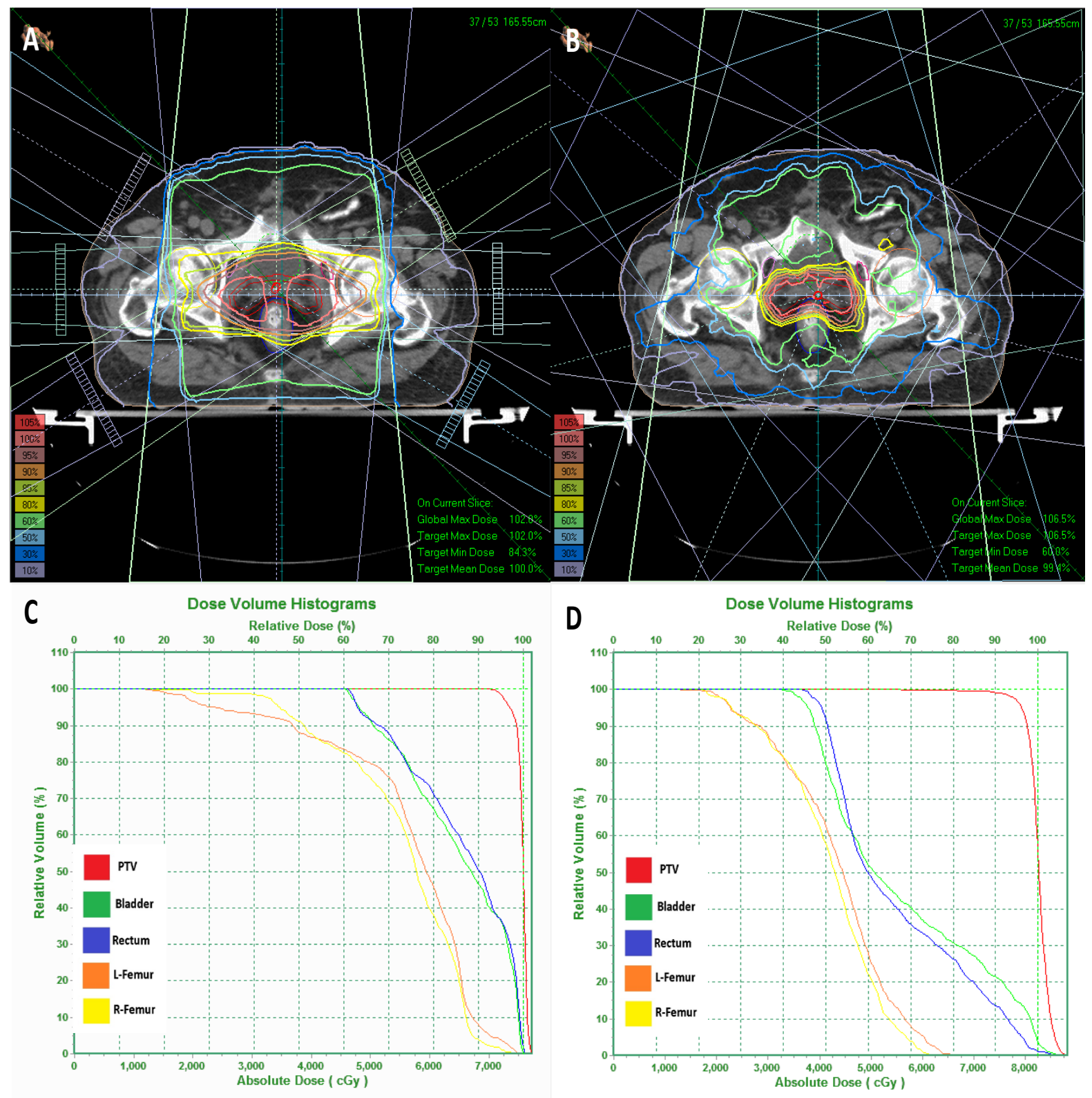

Figure 1: Dose distributions and dose volume histograms of 3D-CRT and IMRT plans for patient \#3. (A) dose distribution for 3D-CRT. (B)dose distribution for IMRT.(C) dose-volume histogram for 3D-CRT. (D) dose-volume histogram for IMRT. 
Table 3: NTCP\% for rectum considering two different degree of complications including (proctitis/necrosis/stenosis/fistula) and Mild RTOG2/3 complication for both plans.

\section{Complication (proctitis/necrosis/stenosis/fistula) Mild RTOG2/3 complication}

\begin{tabular}{ccccc}
\hline Patient & 3D-CRT & IMRT & 3D-CRT & IMRT \\
\hline $\mathbf{1}$ & 10.3 & 8.1 & 96.8 & 89.9 \\
\hline $\mathbf{2}$ & 8.5 & 6.7 & 90.1 & 51.3 \\
\hline $\mathbf{3}$ & 11.2 & 5.9 & 98.3 & 49.4 \\
\hline $\mathbf{4}$ & 7.1 & 6.9 & 83.7 & 58.7 \\
\hline $\mathbf{5}$ & 3.9 & 3.3 & 19.2 & 7.7 \\
\hline $\mathbf{6}$ & 6.9 & 6.1 & 79.5 & 50.9 \\
\hline $\mathbf{7}$ & 10.6 & 7.9 & 95.4 & 60.7 \\
\hline $\mathbf{8}$ & 12 & 10 & 98.8 & 82.5 \\
\hline $\mathbf{9}$ & 10.5 & 10.5 & 95.8 & 82.4 \\
\hline $\mathbf{1 0}$ & 7.2 & 5.2 & 75.3 & 36.4 \\
\hline Average & 8.8 & 7.06 & 83.29 & 56.99 \\
\hline SD & 2.52 & 2.16 & 23.99 & 24.44 \\
\hline P value & 0.005 & & 0.0002 &
\end{tabular}

results indicated that there was a statistically significant difference $(\mathrm{P}$-value $<0.005)$ between two treatment techniques considering both complications.

NTCP for bladder is tabulated in Table 4. For both techniques, the complication rate was less than $2 \%$ on average. However, the NTCP for IMRT plans was about 10 times lower than 3D-CRT with average of $0.18 \%$. In addition, the difference was statistically significant $(\mathrm{P}$ value $<0.039$ ).

For femoral heads, the NTCP of about $4.5 \%$ was seen for 3D-CRT plans on average, while for IMRT plans, NTCP of zero was estimated. Paired t-test showed a p-value of 0.01 for the difference between two techniques.

The results of TCP calculations using Poisson model are shown in Table 5. Different $\alpha / \beta$ values have been proposed for prostate cancer in previous studies $[12,21,22]$ and some suggested a value between 1 to 5 , while some used up to 10 in their studies [12]. The comparison was made between 3D-CRT and IMRT for dif-
Table 4: NTCP(\%) for Bladder in 3D-CRT and IMRT (Symptomatic bladder contracture and volume loss).

\begin{tabular}{ccc} 
Complication & \multicolumn{2}{c}{$\begin{array}{c}\text { (Symptomatic bladder } \\
\text { contracture and volume) }\end{array}$} \\
\hline Patient & 3D-CRT & IMRT \\
\hline $\mathbf{1}$ & 2.1 & 1.5 \\
\hline $\mathbf{2}$ & 1 & 0.1 \\
\hline $\mathbf{3}$ & 1.4 & 0.1 \\
\hline $\mathbf{4}$ & 0.3 & 0 \\
\hline $\mathbf{5}$ & 0 & 0 \\
\hline $\mathbf{6}$ & 0.1 & 0 \\
\hline $\mathbf{7}$ & 0.2 & 0.1 \\
\hline $\mathbf{8}$ & 0.9 & 0 \\
\hline $\mathbf{9}$ & 4.6 & 0 \\
\hline $\mathbf{1 0}$ & 1.5 & 0 \\
\hline Average & 1.21 & 0.18 \\
\hline SD & 1.37 & 0.46 \\
\hline P value & 0.039 & \\
\hline
\end{tabular}


Table 5: TCP(\%) for Prostate in 3D-CONFORMAL treatments and intensity modulated radiation therapy using Poisson model

\begin{tabular}{ccccccccc}
$\mathbf{\alpha} / \boldsymbol{\beta}$ & \multicolumn{2}{c}{$\mathbf{1 . 5}$} & \multicolumn{2}{c}{$\mathbf{3}$} & \multicolumn{2}{c}{$\mathbf{5}$} & \multicolumn{2}{c}{$\mathbf{1 0}$} \\
\hline Case No. & 3D & IMRT & 3D & IMRT & 3D & IMRT & 3D & IMRT \\
\hline $\mathbf{1}$ & 100 & 100 & 97.2 & 99 & 90.2 & 94.2 & 77.6 & 85.1 \\
\hline $\mathbf{2}$ & 100 & 100 & 98.3 & 99.3 & 92.5 & 95.2 & 81.7 & 87.2 \\
\hline $\mathbf{3}$ & 100 & 100 & 97.3 & 95.2 & 90.3 & 88.3 & 77.6 & 77.7 \\
\hline $\mathbf{4}$ & 100 & 100 & 97.6 & 98.7 & 91 & 93.9 & 78.8 & 84.7 \\
\hline $\mathbf{5}$ & 100 & 100 & 98 & 99.1 & 91.7 & 94.6 & 80 & 85.9 \\
\hline $\mathbf{6}$ & 100 & 100 & 97.3 & 96.8 & 91.4 & 90.4 & 79.5 & 79.7 \\
\hline $\mathbf{7}$ & 100 & 100 & 97.8 & 96.5 & 91.4 & 90.1 & 79.5 & 79.8 \\
\hline $\mathbf{8}$ & 100 & 100 & 97.2 & 97.3 & 90.2 & 91.5 & 77.6 & 81.7 \\
\hline $\mathbf{9}$ & 100 & 100 & 97.5 & 98.6 & 90.7 & 93.6 & 78.4 & 84.3 \\
\hline $\mathbf{1 0}$ & 100 & 100 & 98.6 & 99.3 & 93.2 & 95.2 & 82.9 & 87.1 \\
\hline Average & 100 & 100 & 97.68 & 97.98 & 91.26 & 92.7 & 79.36 & 83.32 \\
\hline SD & 0 & 0 & 0.48 & 1.43 & 1 & 2.43 & 1.79 & 3.36 \\
\hline P value & & ---- & & 0.46 & & 0.059 & 0.001
\end{tabular}

ferent $\alpha / \beta$ values. As it can be seen, there is no difference in TCP between 3D-CRT and IMRT for the $\alpha / \beta$ of 1.5 in all studied cases. However, the difference between two methods increases with increase in $\alpha / \beta$ and after $\alpha / \beta$ of 5 ; the difference between two methods are statistically significant. The results demonstrated that dose escalation from 76 Gy in 3D-CRT to 82 Gy for IMRT caused 5\% improvement in TCP for prostate cancer considering the $\alpha / \beta$ of 10 .

TCP and EUD were also calculated by EUD model for prostate cancer plans (Table 6). The average EUD of 80.9 Gy and 76.77 Gy were obtained for IMRT and 3D-CRT, respectively. While, there was no significant difference between average TCPs for both methods considering the TCP of $98.5 \%$ for IMRT and $98.16 \%$ for 3D-CRT (for $\alpha / \beta=5,20$ ).

In Table 7, the results of NTCP for rectum are tabulated for 10 prostate cancer patients. In this model, for IMRT plans, the average EUD was about 0.84 Gy less than 3D-CRT plans. However, this difference was not statistically significant ( $p$-value $=0.19$ ). Also, the compar- ison between two methods revealed that the NTCP of rectum for IMRT plans was less than 3D-CRT plans with a p-value of 0.058 .

In Table 8, the NTCP of bladder is tabulated. The results showed that IMRT complication for bladder was about10 times less than that for 3D-CRT. Additionally, the difference between two methods in terms of NTCP and EUD of bladder was significant and statistically meaningful.

The NTCP and EUD were also calculated for both right and left femoral heads. The EUD for IMRT plans (32.92Gy) was 16 Gy lower compared to 3D-CRT plans (48.44) on average for both femoral heads. Additionally, the NTCP for IMRT plans was close to zero for femoral heads, while for 3D-CRT an NTCP of about $2 \%$ was seen.

\section{Discussion}

In the current study, the biological evaluation of ten prostate patients was investigated to find out the effect of dosimetric difference on estimated biological scores of TCP and NTCP. 
Table 6: EUD(Gy) and TCP(\%) estimations for prostate cancer patients in 3D-CRT and IMRT techniques using EUD model

\begin{tabular}{|c|c|c|c|c|c|c|c|c|c|c|c|c|}
\hline \multirow{3}{*}{$\begin{array}{c}\alpha / \beta \\
\text { Case } \\
\text { No. }\end{array}$} & \multicolumn{4}{|c|}{1.5} & \multicolumn{4}{|c|}{5} & \multicolumn{4}{|c|}{20} \\
\hline & \multicolumn{2}{|c|}{ EUD } & \multicolumn{2}{|c|}{ TCP } & \multicolumn{2}{|c|}{ EUD } & \multicolumn{2}{|c|}{ TCP } & \multicolumn{2}{|c|}{ EUD } & \multicolumn{2}{|c|}{ TCP } \\
\hline & 3D & IMRT & $3 D$ & IMRT & $3 D$ & IMRT & $3 D$ & IMRT & $3 \mathrm{D}$ & IMRT & $3 D$ & IMRT \\
\hline 1 & 77.05 & 80.62 & 98.20 & 98.49 & 76.86 & 80.94 & 98.18 & 98.51 & 76.73 & 81.12 & 98.17 & 98.53 \\
\hline 2 & 76.92 & 82.25 & 98.19 & 98.61 & 76.76 & 82.31 & 98.17 & 98.61 & 76.66 & 82.32 & 98.16 & 98.61 \\
\hline 3 & 75.21 & 79.18 & 98.02 & 98.38 & 75.39 & 80.98 & 98.04 & 98.52 & 75.49 & 81.56 & 98.05 & 98.56 \\
\hline 4 & 76.44 & 81.89 & 98.14 & 98.58 & 76.44 & 82.06 & 98.14 & 98.59 & 76.41 & 82.12 & 98.14 & 98.60 \\
\hline 5 & 76.80 & 82.25 & 98.18 & 98.61 & 76.68 & 82.31 & 98.16 & 98.61 & 76.58 & 82.31 & 98.15 & 98.61 \\
\hline 6 & 76.24 & 79.48 & 96.12 & 98.40 & 76.30 & 80.77 & 98.13 & 98.50 & 76.30 & 81.32 & 98.13 & 98.54 \\
\hline 7 & 77.72 & 80.00 & 98.26 & 98.45 & 77.45 & 81.14 & 98.23 & 98.53 & 77.25 & 81.55 & 98.22 & 98.56 \\
\hline 8 & 75.81 & 80.83 & 98.08 & 98.51 & 75.96 & 81.67 & 98.09 & 98.57 & 76.02 & 81.96 & 98.10 & 98.59 \\
\hline 9 & 76.85 & 81.89 & 98.18 & 98.58 & 76.80 & 82.12 & 98.18 & 98.60 & 76.73 & 82.19 & 98.17 & 98.60 \\
\hline 10 & 78.52 & 82.37 & 98.33 & 98.61 & 78.08 & 82.43 & 98.29 & 98.62 & 77.78 & 82.42 & 98.26 & 98.62 \\
\hline Average & 76.75 & 81.07 & 97.97 & 98.52 & 76.67 & 81.67 & 98.16 & 98.56 & 76.59 & 81.88 & 98.15 & 98.58 \\
\hline SD & 0.93 & 1.21 & 0.65 & 0.08 & 0.74 & 0.65 & 0.06 & 0.04 & 0.62 & 0.46 & 0.05 & 0.03 \\
\hline$P$ value & & & & & & & & & & & & \\
\hline
\end{tabular}

Based on our results, the NTCPs of rectum and bladder were calculated by LKB model using Bioplan software. It was found that for rectum, the average dose for all patients was about 7.22 Gy lower for IMRT compared to 3D-CRT. However, if we consider the resultant complication probability for proctititis/ necrosis/fistula, the NTCP for IMRT plans $(7.06 \%)$ was almost $20 \%$ less than for 3DCRT (8.8\%). In other words, $7.22 \mathrm{~Gy}$ reduction in mean dose leads to $20 \%$ lower complication for IMRT plans. Considering mild RTOG2/3 complication for rectum, the estimated NTCPs indicated that most of the patients will experience this complication with about 10 times higher probability compared to proctitis/stenosis/fistula complication. For IMRT plans, this complication probability was estimated $32 \%$ less relative to 3D-CRT plans. Lower average dose of rectum in IMRT plans relative to 3D-CRT plans was responsible for reduced NTCP in IMRT plans, and it stems from fact that the maximum received dose by anterior $1 / 3$ part of rectum was 6 Gy higher in IMRT plans, but the posterior volume of rectum received lower dose in IMRT plans relative to 3D-CRT plans. It should also be reminded that in 3D-CRT plans, the dose of 50 Gy is delivered to whole pelvis using two wide parallel opposed anterior-posterior fields. Consequently, the whole volume of rectum receives this dose completely, while in IMRT there was a great possibility to reduce the delivered dose in distant part of rectum using intensity modulated beams.

Our results were in accordance with the study of Cambria et al. in which they analyzed 3D-CRT plans of 57 patients to validate the reliability of LKB model. They compared NTCP results with clinical complications of late rectal toxicity and demonstrated an agreement between the toxicity rate evaluated by DVH constraints, by the LKB model and the clinical outcome [11]. Moreover, our results can be compared with the study of Luxton et al. which used LKB model for NTCP calculations of IMRT and 3D-CRT plans [3]. Our average NTCP for bladder was in close agree- 
Table 7: EUD(Gy) \& NTCP(\%) for Rectum in 3D-CRT and IMRT

\begin{tabular}{ccccc}
\multicolumn{2}{c}{ EUD (Gy) } & \multicolumn{2}{c}{ NTCP(\%) } \\
\hline Patient & 3D-CRT & IMRT & 3D-CRT & IMRT \\
\hline $\mathbf{1}$ & 69.18 & 67.57 & 8.92 & 6.29 \\
\hline $\mathbf{2}$ & 66.1 & 64.35 & 4.51 & 2.98 \\
\hline $\mathbf{3}$ & 68.05 & 63.6 & 6.99 & 2.48 \\
\hline $\mathbf{4}$ & 66.54 & 66.31 & 4.99 & 4.73 \\
\hline $\mathbf{5}$ & 59.01 & 61.2 & 0.76 & 1.35 \\
\hline $\mathbf{6}$ & 64.69 & 66.02 & 3.23 & 4.42 \\
\hline $\mathbf{7}$ & 67.59 & 65.39 & 6.32 & 3.82 \\
\hline $\mathbf{8}$ & 68.57 & 67.02 & 7.82 & 5.56 \\
\hline $\mathbf{9}$ & 67.52 & 67.33 & 6.22 & 5.96 \\
\hline $\mathbf{1 0}$ & 64.9 & 64.96 & 3.4 & 3.45 \\
\hline Average & 66.21 & 65.37 & 5.31 & 4.10 \\
\hline SD & 2.93 & 1.95 & 2.43 & 1.59 \\
\hline P value & 0.19 & & 0.058 & \\
\hline
\end{tabular}

Table 8: EUD(Gy) \& NTCP(\%) for Bladder in 3D-CRT and IMRT

\begin{tabular}{ccccc} 
& \multicolumn{2}{c}{ EUD (Gy) } & \multicolumn{2}{c}{ NTCP(\%) } \\
\hline Patient & 3D-CRT & IMRT & 3D-CRT & IMRT \\
\hline $\mathbf{1}$ & 63.17 & 54.22 & 2.23 & 0.19 \\
\hline $\mathbf{2}$ & 64.68 & 55.64 & 3.22 & 0.29 \\
\hline $\mathbf{3}$ & 64.24 & 55.93 & 2.90 & 0.32 \\
\hline $\mathbf{4}$ & 58.72 & 53.89 & 0.7 & 0.17 \\
\hline $\mathbf{5}$ & 53.56 & 52.25 & 0.16 & 0.1 \\
\hline $\mathbf{6}$ & 55.17 & 53.09 & 0.26 & 0.14 \\
\hline $\mathbf{7}$ & 58.79 & 55.78 & 0.71 & 0.31 \\
\hline $\mathbf{8}$ & 62.86 & 54.00 & 2.07 & 0.18 \\
\hline $\mathbf{9}$ & 61.47 & 51.55 & 1.45 & 0.08 \\
\hline $\mathbf{1 0}$ & 64.54 & 53.67 & 3.12 & 0.16 \\
\hline Average & 60.72 & 54.00 & 1.68 & 0.194 \\
\hline SD & 3.99 & 1.47 & 1.18 & 0.08 \\
\hline P value & 0.0002 & & 0.002 & \\
\hline
\end{tabular}


ment with their data, while for rectum complication, our NTCP was considerably higher because of the differences in parameters used for LKB model, treatment planning, patients' stage, etc. However, our NTCP for rectum was in agreement with the results of Hancock et al. where they reported NTCP of $60 \%$ and $17 \%$ for IMRT and 3D-CRT, respectively for acute RTOG grade 2 toxicity [23].

The NTCP estimation for bladder using LKB model showed that the probability of symptomatic bladder contracture was $5 \%$ in its worst case for a 3D-CRT treatment. Additionally, the average NTCP indicated 6.7 times higher complication for 3D-CRT compared to IMRT plans. It should be mentioned here that the superiority of IMRT relative to 3D-CRT was more prominent considering the bladder complication probabilities. Besides, averaged values for mean dose of bladder were 54.08 Gy and 62.63 Gy for IMRT and 3D-CRT plans, respectively (8.55 Gy less dose for IMRT).

The results of NTCP for bladder and rectum in two techniques indicate a lower NTCP for bladder compared to rectum as well as negligible complication for IMRT plans (around 1\%). Considering very close mean doses of both organs in the studied plans, the observed difference could be attributed to different $m$ and $n$ parameters used for NTCP calculation in LKB model. It is why, higher $m$ value will increase the NTCP, and inversely lower $n$ value leads to higher NTCP for every organ. Comparing the $\mathrm{m}$ and $\mathrm{n}$ values for rectum and bladder shows higher $\mathrm{m}$ and lower $\mathrm{n}$ for rectum relative to bladder, thus, the NTCP for rectum will be higher than the bladder.

For mild rectum complication, the $m$ value was lower than proctitis/necrosis complication, but TD50 was significantly lower (56 Gy vs. 80 Gy), and it caused higher NTCP estimation for mild rectum complication.

NTCP estimations for both femoral heads showed $0 \%$ for IMRT plans compared to $4.5 \%$ for 3D-CRT plans, while the difference in mean doses of femoral heads between 3D-
CRT and IMRT plans were about 8 Gy (49 Gy vs. 41 Gy).

NTCPs of all studied organs were also calculated by EUD model. In this model, the proctitis probability was calculated for rectum, and it was lower than LKB model for both 3DCRT and IMRT techniques (4.1\% for EUD vs.7.06\% for Bioplan for IMRT plans). For bladder and femoral heads, the results of EUD model were slightly higher but very similar to the Bioplan LKB model results.

The results of TCP calculations using Bioplan software indicated this fact that TCP was very dependent on $\alpha / \beta$ ratio. Additionally, with increase in $\alpha / \beta$ ratio from 1.5 to 20 , TCP decreased and the differences between calculated TCP for 3D-CRT and IMRT were augmented. The TCP of IMRT for $\alpha / \beta$ of 10 which was used by some previous studies was 5\% higher than 3D-CRT. TCP was also calculated using EUD model to show a negligible difference between 3D-CRT and IMRT plans; it was very similar to the results of Bioplan with $\alpha / \beta$ of 3 . The important point is that the TCP results by Bioplan using Poisson model differed considerably with $\alpha / \beta$ ratio, where in EUD the variation of TCP with $\alpha / \beta$ ratio was small. TCP estimation by Bioplan and its dependency on $\alpha / \beta$ was very similar to the results of Deb and Fielding [12]. They reported that lower $\alpha / \beta$ leads to higher TCP in all studied cases of prostate cancer. However, they used Kallman S-model which was available on Pinnacle TPS. In our study, NTCP for rectum was higher than bladder, and the same results were reported in their study. Also, our EUD-calculated TCP were very close to their obtained TCP of around $98 \%$ in their study.

Another important point that should be noted here is the patient-by-patient variations of NTCPs which originate from the differences in PTV volume for each patient. Koontz et al. conducted a radiobiological modelling of IMRT vs. 3D conformal planning for postprostatectomy radiotherapy cases [2]. They found that in spite of significant dosimetric 
differences between two techniques, no significant difference was found in the NTCP for either bladder or rectal injury. Also, they concluded that due to relatively low doses given to the bladder and rectum, no statistically significant improvement was found in NTCP between the 3D conformal and IMRT plans. Our results differed significantly from their finding because our plans were performed on intact prostate cancers with larger PTV which lead to higher dose given to both rectum and bladder. It shows that the obtained NTCPs of bladder and rectum are dependent on the volume of PTV. Moreover, our results indicated that for patients with larger PTV, the obtained NTCPs for rectum and bladder were higher.

\section{Conclusion}

This study investigated the use of different radiobiological models for comparison of 3DCRT and IMRT plans of prostate cancer. Our results demonstrated that IMRT plans produce significantly lower NTCP for rectum, bladder and femoral heads in both LKB- and EUDbased radiobiological models. In contrast, for TCP calculations, there was no significant gain with IMRT plans compared to 3D-CRT plans using EUD model. On the other hand, TCP estimation was different for Poisson and EUD model. EUD-based TCP showed less dependency on $\alpha / \beta$ parameter, while for Poisson model, the TCP estimation varied considerably with $\alpha / \beta$.

Our study confirmed the reliability of our new TPS calculations using dosimetric comparisons as well as radiobiological modelling which was a more clinically significant method. Different TCP estimations for prostate cancer and its dependency on used radiobiological model highlight the need for more clinical investigation in order to gather and analyze follow-up data for radiobiological model validation.

\section{Acknowledgment}

This project was conducted as a M.Sc. the- sis No.93/2-7/12 of medical school registered and financially supported by Immunology Research Center of Tabriz University of Medical Sciences. The authors would like to thank Radiation Oncology department of Tabriz International Hospital for their valuable contribution to this research.

\section{Conflict of Interest}

None

\section{References}

1. Jensen I, Carl J, Lund B, Larsen EH, Nielsen J. Radiobiological impact of reduced margins and treatment technique for prostate cancer in terms of tumor control probability (TCP) and normal tissue complication probability (NTCP). Med Dosim. 2011;36:130-7. doi.org/10.1016/j.meddos.2010.02.004. PubMed PMID: 20488692.

2. Koontz BF, Das S, Temple K, Bynum S, Catalano $\mathrm{S}$, Koontz $\mathrm{Jl}$, et al. Dosimetric and radiobiologic comparison of 3D conformal versus intensity modulated planning techniques for prostate bed radiotherapy. Med Dosim. 2009;34:256-60. doi. org/10.1016/j.meddos.2008.10.005. PubMed PMID: 19647638.

3. Luxton G, Hancock SL, Boyer AL. Dosimetry and radiobiologic model comparison of IMRT and 3D conformal radiotherapy in treatment of carcinoma of the prostate. Int $J$ Radiat Oncol Biol Phys. 2004;59:267-84. doi.org/10.1016/j. ijrobp.2004.01.024. PubMed PMID: 15093924.

4. Nahum AE, Uzan J. (Radio)biological optimization of external-beam radiotherapy. Comput Math Methods Med. 2012;2012:329214. PubMed PMID: 23251227. PubMed PMCID: 3508750.

5. Rana S, Cheng $C$. Radiobiological impact of planning techniques for prostate cancer in terms of tumor control probability and normal tissue complication probability. Ann Med Health Sci Res. 2014;4:16772. doi.org/10.4103/2141-9248.129023. PubMed PMID: 24761232. PubMed PMCID: 3991934.

6. Niemierko A, Goitein M. Calculation of normal tissue complication probability and dose-volume histogram reduction schemes for tissues with a critical element architecture. Radiother Oncol. 1991;20:166-76. doi.org/10.1016/01678140(91)90093-V. PubMed PMID: 1852908.

7. Stavreva N, Niemierko A, Stavrev P, Goitein M. Modelling the dose-volume response of the spinal cord, based on the idea of damage to contiguous functional subunits. Int J Radiat Biol. 2001;77:695- 
702. doi.org/10.1080/09553000110047555. PubMed PMID: 11403709.

8. Warkentin B, Stavrev P, Stavreva N, Field C, FalIone BG. A TCP-NTCP estimation module using DVHs and known radiobiological models and parameter sets. J Appl Clin Med Phys. 2004;5:50-63. doi.org/10.1120/jacmp.v5i1.1970. PubMed PMID: 15753933.

9. Ahmad S, Vogds BJ, McKenna F, Vlachaki MT. Tumor control probability (TCP) in prostate cancer: role of radiobiological parameters and radiation dose escalation. J Xray Sci Technol. 2009;17:34754. PubMed PMID: 19923690.

10. Krupa P, Ticha H, Kazda T, Dymackova R, Zitterbartova J, Odlozilikova A, et al. Early toxicity of hypofractionated radiotherapy for prostate cancer. Biomed Pap Med Fac Univ Palacky Olomouc Czech Repub. 2016;160:435-41. doi.org/10.5507/ bp.2016.008. PubMed PMID: 26948031.

11. Cambria R, Jereczek-Fossa BA, Cattani F, Garibaldi $C$, Zerini $D$, Fodor $C$, et al. Evaluation of late rectal toxicity after conformal radiotherapy for prostate cancer: a comparison between dose-volume constraints and NTCP use. Strahlenther Onkol. 2009;185:384-9. doi.org/10.1007/s00066-0091933-8. PubMed PMID: 19506822.

12. Deb P, Fielding A. Radiobiological model comparison of 3D conformal radiotherapy and IMRT plans for the treatment of prostate cancer. Australas Phys Eng Sci Med. 2009;32:51-61. doi.org/10.1007/ BF03178629. PubMed PMID: 19623855.

13. Rancati T, Fiorino C, Gagliardi G, Cattaneo GM, Sanguineti $G$, Borca VC, et al. Fitting late rectal bleeding data using different NTCP models: results from an Italian multi-centric study (AIROPROS0101). Radiother Oncol. 2004;73:21-32. doi.org/10.1016/j.radonc.2004.08.013. PubMed PMID: 15465142.

14. Warren S, Partridge $M$, Carrington $R$, Hurt $C$, Crosby T, Hawkins MA. Radiobiological determination of dose escalation and normal tissue toxicity in definitive chemoradiation therapy for esophageal cancer. Int J Radiat Oncol Biol Phys. 2014;90:4239. doi.org/10.1016/j.jirobp.2014.06.028. PubMed PMID: 25304796. PubMed PMCID: 4165721.

15. Mesbahi A, Zergoug I. Dose Calculations for Lung Inhomogeneity in High-Energy Photon Beams and Small Beamlets: A Comparison between $\mathrm{XiO}$ and
TiGRT Treatment Planning Systems and MCNPX Monte Carlo Code. Iranian Journal of Medical Physics. 2015;12:167-77.

16. Mesbahi A, Dadgar H. Dose calculations accuracy of TiGRT treatment planning system for small IMRT beamlets in heterogeneous lung phantom. International Journal of Radiation Research. 2015;13:345-54.

17. Sanchez-Nieto B, Nahum AE. BIOPLAN: software for the biological evaluation of. Radiotherapy treatment plans. Med Dosim. 2000;25:71-6. doi. org/10.1016/S0958-3947(00)00031-5. PubMed PMID: 10856684.

18. Burman C, Kutcher GJ, Emami B, Goitein M. Fitting of normal tissue tolerance data to an analytic function. Int J Radiat Oncol Biol Phys. 1991;21:123-35. doi.org/10.1016/0360-3016(91)90172-Z. PubMed PMID: 2032883.

19. Lyman JT. Complication probability as assessed from dose-volume histograms. Radiat Res Suppl. 1985;8:S13-9. doi.org/10.2307/3583506. PubMed PMID: 3867079.

20. Gay HA, Niemierko A. A free program for calculating EUD-based NTCP and TCP in external beam radiotherapy. Phys Med. 2007;23:115-25. doi. org/10.1016/j.ejmp.2007.07.001. PubMed PMID: 17825595.

21. Carlone $M$, Wilkins $D$, Nyiri $B$, Raaphorst $P$. Comparison of alpha/beta estimates from homogeneous (individual) and heterogeneous (population) tumor control models for early stage prostate cancer. Med Phys. 2003;30:2832-48. doi.org/10.1118/1.1612946. PubMed PMID: 14596319.

22. Valdagni $R$, Italia $C$, Montanaro $P$, Lanceni $A$, Lattuada $P$, Magnani $T$, et al. Is the alpha-beta ratio of prostate cancer really low? A prospective, nonrandomized trial comparing standard and hyperfractionated conformal radiation therapy. Radiother Oncol. 2005;75:74-82. doi.org/10.1016/j. radonc.2004.12.019. PubMed PMID: 15878104.

23. Hancock S, Luxton G, Chen Y, Xing L, Boyer A. Intensity modulated radiotherapy for localized or regional treatment of prostatic cancer: Clinical implementation and improvement in acute tolerance. International Journal of Radiation Oncology * Biology* Physics. 2000;48:252-3. doi.org/10.1016/ S0360-3016(00)80301-6. 